\title{
Enhanced Graphene Sensors via Multi-lasing Fabrication
}

\author{
A. Kaidarova, M.T Vijjapu, K.Telegenov, A.Przybysz, K.N. Salama, and J. Kosel
}

\begin{abstract}
Resistive strain and bending sensors offer a versatile platform for sensing various physical parameters with relatively little effort and budget. The lightweight, robust and compact sensors are extensively used in manifold low-power applications. Recently, scribed and flexible laserinduced graphene sensors have shown potent capabilities for a variety of measurements, including flow, deflection, and force. Achieving a high sensitivity to various stimuli remains a challenge due to limited change in relative resistance. In this paper, we report a multifunctional LIG sensor with widely tunable properties and significantly enhanced electromechanical performance. A method of repeated laser writing is used to increase the porosity, the uniform carbonization degree and, most importantly, the sensitivity of the LIG sensors. A gauge factor of

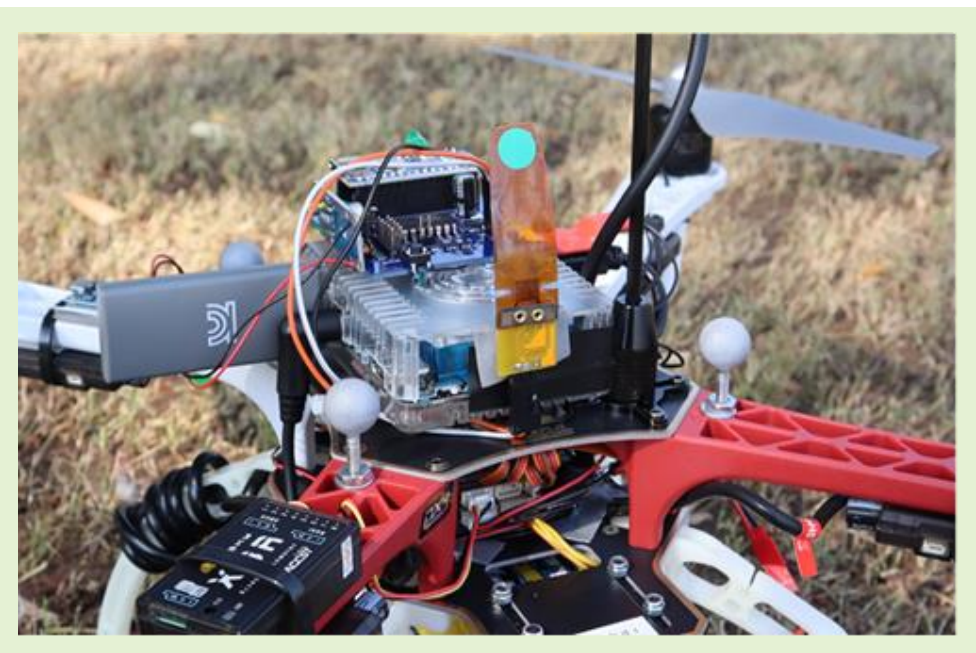
91.2 is achieved after three-times laser writing at low power, which is an increase of $750 \%$ to one-time laser writing and $720 \%$ higher than the ones previously reported for LIG strain sensors. The increase is attributed to a more porous surface morphology that provides more overlapping area and displacement of the graphene layers. A homogeneous bidirectional response was obtained by scribing the electrodes on both faces of the substrate. Parylene C-coating is used to protect the LIG sensors from environmental effects. Coated sensors were packaged to a PCB assembly for easy integration into various applications. An example is a LIG bending sensor customized for velocity profile monitoring of Unmanned Aerial Vehicles in the outdoor environment.
\end{abstract}

Index Terms - flexible, graphene, sensors, strain, unmanned aerial vehicles, velocity.

\section{Introduction}

$T^{1}$ HE potential and versatility of bending sensors made of porous graphene were recently demonstrated for an extensive range of configurations, including measurements of force, curvature, and speed [1-5]. The deflection caused by a wide range of forces and speeds was effectively sensed through a resistance measurement, exploiting the piezoresistance of the scribed graphene films $[2,3]$. The graphene electrodes were realized using flexible and lightweight polyimide (PI) films and a simple laser scribing process, creating what is known as Laser-Induced Graphene (LIG) [6]. This facile fabrication process is a combination of large-area graphene growth and patterning

A. Kaidarova, M.T. Vijjapu, K. Telegenov, A.Przybysz, K. N. Salama were with the Computer, Electrical and Mathematical Sciences \& Engineering (CEMSE) department, King Abdullah University of Science and Technology (KAUST), 23955, Thuwal, Kingdom of Saudi Arabia 2395 (altynay.kaidarova@kaust.edu.sa).

J. Shamma was with the Grainger College of Engineering, University of Illionois, Urbana, IL 61801-3080

J.Kosel was with Sensor Systems Division, Silicon Austria Labs (SAL), High Tech Campus, Europastraße 12, A-9524 Villach, Austria in one step, without using any high-cost cleanroom facilities, wet chemical processes, and consecutive treatments [5]. Besides these advantages, LIG is also corrosion-resistant [7-9] and can operate at high temperatures of up to $580^{\circ}[5,10]$. Thus, LIG has shown its suitability to be utilized in several physical domains. The mechanical flexibility of LIG bending sensors paved the way for low-cost electromechanical measurement systems, such as body movements tracking ${ }^{[1,4,11-13]}$, marine animals speed monitoring [2], and sensing the surface current velocity of the sea ${ }^{[2]}$. Meanwhile, LIG heaters based on the Joule effect were used in broad applications ranging from self-sterilizing filters ${ }^{[10]}$ to gas sensors ${ }^{[14}$, 15]. For example, thermally actuated LIG films were unlisted to capture and kill bacteria, and molecules that may cause negative reaction and/or illness ${ }^{[10]}$. Self-heated gas sensors obtained by dispersing nanomaterials with different selectivity on porous LIG surface were used for deconvolution of various gaseous components in the mixture ${ }^{[14]}$. The exploitation of LIG in the microwave, radio wave ${ }^{[16]}$ and terahertz regimes ${ }^{[17]}$ and in solar-tothermal ${ }^{[18]}$ conversion applications has been reported as well. Recently, Zhu et al., demonstrated the possibility to 
progress from individual LIG devices to all-LIG integrated systems by combining wireless transmission, energy harvesting modules and stretchable sensors into a single platform ${ }^{[15]}$. Due to the coupled mechanicselectromagnetic design, the wideband dipole antenna showed deformation-independent radiation properties to be used in self-powered systems, remote monitoring of the environment, and clean energy applications ${ }^{[15]}$. Although the sensitivity and dynamic range of the sensors could be tuned via the sensor geometry, the overall resistance variation was limited to a maximum of $10 \%$ and a gauge factor of $\sim 11{ }^{[5]}$ A larger change in the value of resistance for a small change in strain would allow more accurate and subtle detection of deflection, force, and curvature, which is crucial in many automotive, industrial, and medical applications. The electromechanical performance of LIG has previously been enhanced by increasing the laser power within a certain range ${ }^{[6,19,20]}$ or by transferring the LIG pattern to an elastomeric substrate, such as polydimethylsiloxane ${ }^{[11,12,21]}$, silicon rubber ${ }^{[22,23]}$, or polystyrene $[13,24]$. The former often causes graphene detachment from the substrate or thermal damage, resulting in quick performance degradation and unreliable measurements. The latter involves multi-step fabrication processes, which is neither an efficient nor cost-effective way of producing high-volume end products. Moreover, the labor involved in transferring LIG to other substrates slows down the fabrication process compared to fully automated methods.

In this manuscript, we report a fast, scalable fabrication of multifunctional LIG bending sensors with widely tunable properties and significantly enhanced electromechanical performance. We study the effects of repeated laser writing, which resulted in the increase of porosity, while

a)

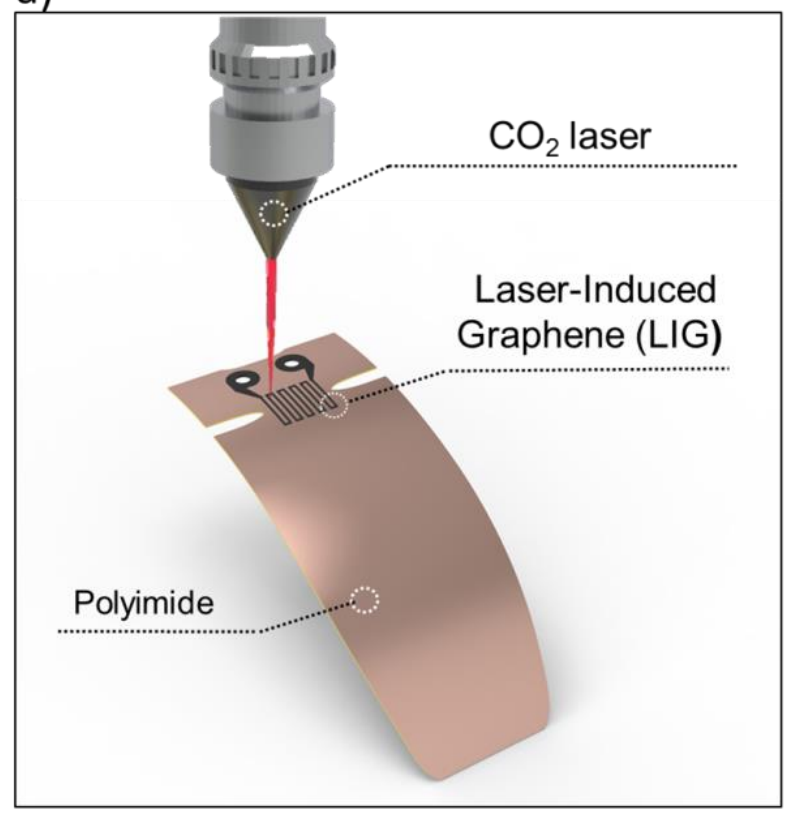

reducing intrinsic structural defects. The sensor design is also customized to achieve a homogeneous bidirectional response. To easily integrate the LIG sensor into various applications, an expandable, plug-and-play sensor module was developed and utilized in velocity profile monitoring of Unmanned Aerial Vehicles (UAV).

Velocity updates of UAVs greatly enhance the navigation process, as the horizontal velocity is coupled with their roll and pitch angles. ${ }^{[25]}$ The key component to evaluate the position and velocity of the drone is the Inertial Measurement Unit (IMU), assembled from accelerometers, gyroscopes, and magnetometers. ${ }^{[26]}$ Typically, the IMU alone provides a reliable state estimation solution, but the error will accumulate over time due to the mathematical integration of signals. ${ }^{[27-29]}$ Particularly, IMUs that are built using MEMs techniques inevitably include time-varying bias, cross-coupling errors, random noise, resulting in error accumulation and signal drifting. ${ }^{[30-32]}$ To overcome this issue, the global navigation satellite systems (GNSS), which provide absolute position and velocity updates, have been utilized. ${ }^{[33]}$ However, the GNSS support is not always available or reliable in confined areas, such as cities, forests, urban and indoor environments. ${ }^{[34]}$ To obtain a long-term accurate navigation solution, at least one type of absolute update is required. Therefore, various navigation assistance methods were attempted based on technologies such as cameras, Light Detection and Ranging (LIDAR) ${ }^{[35]}$, and Radio Detection and Ranging (RADAR). ${ }^{[36]}$ These techniques suffer from either being unable to operate in dark and featureless areas, high price, heavyweight, high power consumption, and high computational power. In this study, we use a LIG bending sensor to provide absolute velocity updates of UAVs in a real-life environment. b)

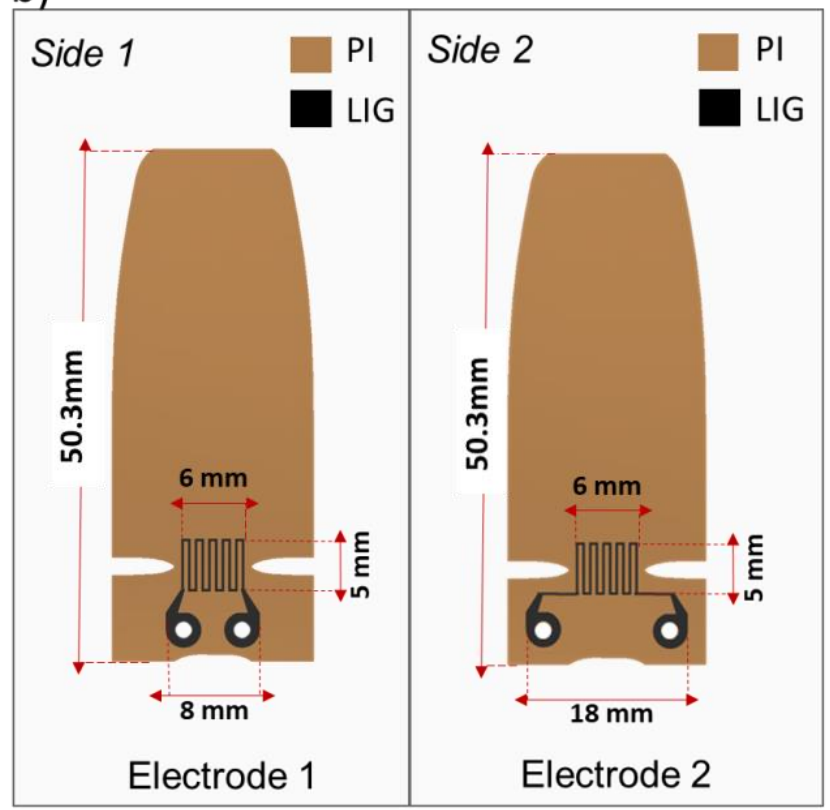

Fig.1 a) Schematic of the fabrication process of LIG sensors; b) Design of double-sided LIG bending sensor; 


\section{METHODS}

\section{A. Fabrication and operation principle}

The sensors were realized by direct and repeated laser scribing on a polyimide (PI) substrate of $127 \mu \mathrm{m}$ (DuPont, Kapton \#IM301449) utilizing pulses from a $\mathrm{CO}_{2}$ laser (ULS, PLS6.75) in ambient conditions, as illustrated in Fig. 1a. The PI surface was cleaned with ethanol and transformed to a $3 \mathrm{D}$ porous graphene film via a laser photothermal process associated with rapid decomposition of gaseous products, the conversion of the available $\mathrm{sp}^{3}$ bonds to $\mathrm{sp}^{2}$ bonds, and an increase in electrical conductivity. ${ }^{[37-39]}$ The following laser parameters were utilized for each iteration of repeated laser writing: $3.5 \mathrm{~W}$ power, $2.9 \mathrm{~cm} / \mathrm{s}$ speed, 1000 pulses per inch (PPI), and 6 $\mathrm{mm}$ working distance. The samples with a maximum of 5 iterations of repeated laser writing were scribed. The versatile fabrication process allowed fabricating a doublesided LIG electrode with optimized geometry and size, as shown in Fig. 1b. Both electrodes were short in length $(1=$ $5 \mathrm{~mm}$ ) and structured in a meander shape (number of turns $=9)$ to achieve a large variation in resistance with the smallest electrode width $(\sim 60 \mu \mathrm{m})$. The working mechanism of bending LIG sensors has already been described in detail elsewhere. [2] In brief, the bending sensor uses porous graphene on a pliable PI substrate to transduce a signal through cantilever deflection, which is anchored at only one end. When a sensor is exposed to external forces, such as airflow, cantilever bending stimulates a fractional resistance variation. The LIG sensors scribed on both sides of the PI substrate can be a)

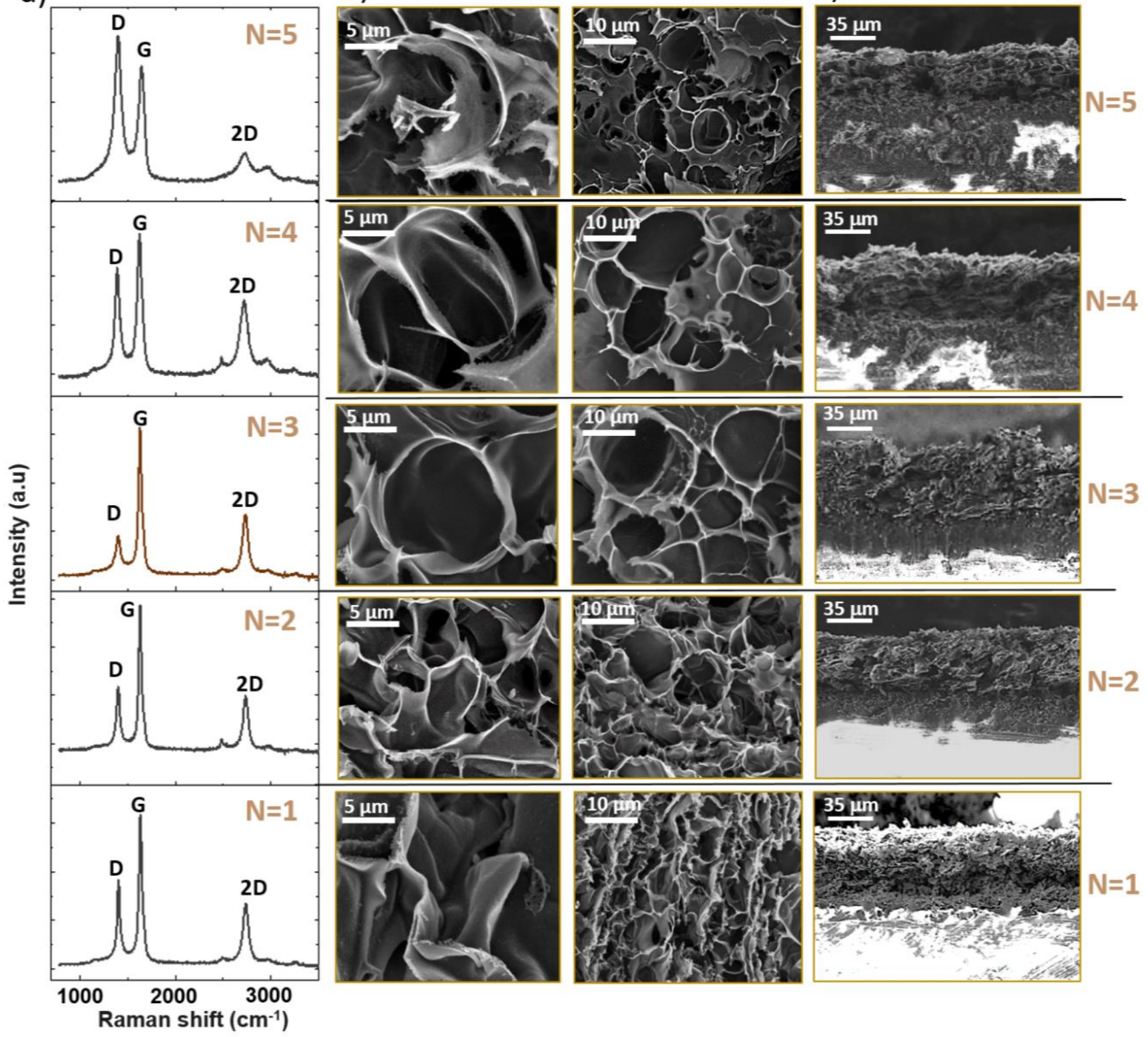

c)

Fig. 2 a) Raman spectrum of LIG for five consecutive laser irradiations. b) Top-view and c) cross-sectional SEM images of porous graphene structures after each laser irradiation step. 
effectively utilized for a difference measurement, which subtracts outputs of the sensor generated in compression and tension states.

\section{B. Characterization techniques.}

The confocal Raman spectroscopy (Alpha 300 Apron, Witec), Scanning Electron Microscopy (SEM, Nova Nano 630 Systems), X-ray photoelectron spectroscopy (XPS, ESCA 3400, Amicus Kratos Analytical), and X-ray diffraction (XRD, D2 Phaser, Bruker) were utilized to investigate the degree of graphitization and defect formation for various LIG samples after each laser writing stage. Fig. 2a shows Raman spectra obtained with an excitation laser source of $532 \mathrm{~nm}$ under $5 \mathrm{~mW}$ power. Three distinct peaks at around $1360 \mathrm{~cm}-1$ (D-band), 1584 $\mathrm{cm}^{-1}$ (G-band), and $2730 \mathrm{~cm}-1$ (2D-band) can be identified even after four times repeated lasing, which is indicative of LIG formation. ${ }^{[40-42]}$ Since the G-band corresponds to the stretching vibration of the sp2 carbon lattice and the D-band to the defects and disorder, the ratio of their intensity (ID/IG) is commonly used to quantify the amount of defects in the graphitic materials. ${ }^{[43]}$ In our case, the ratio ID/IG decreases from 0.79 to 0.26 after three-time

a)
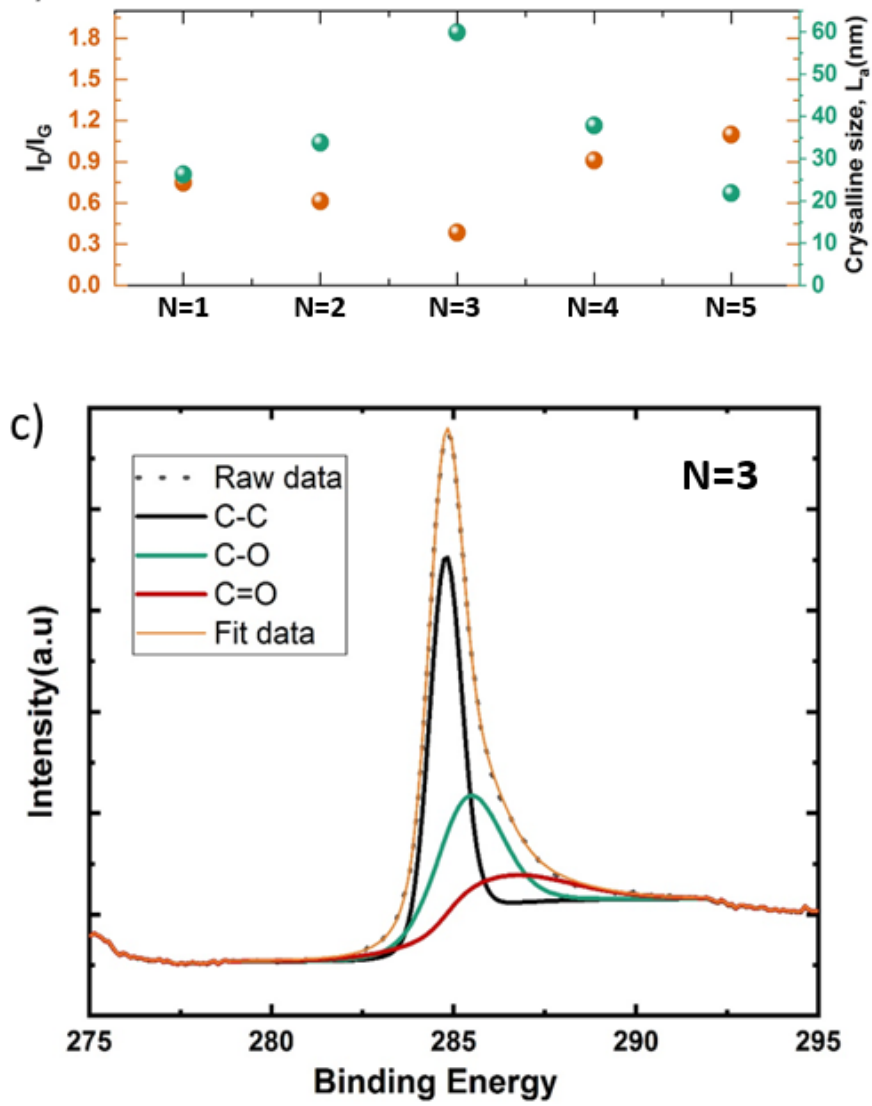

repeated laser writing, indicating reduced structural defects, lower edge densities, and higher quality of the obtained graphene. These results are further verified by SEM images, which show more polyporous and looser surface morphologies obtained after three repeated laser scribing steps (Fig. 2b). The higher porosity observed suggests more air and less LIG in the same volume. Indeed, the weight of the LIG sample decreased by $35 \%$ after three laser writings (Table SI). The consecutive laser writing (four and fivetimes), however, introduces damages to the LIG structure and non-uniform graphene distribution across the PI thickness (Fig. 2c), which is consistent with an increased intensity of the D- band in the Raman spectra. Meanwhile, the $2 \mathrm{D}$ band is responsive to the order of the graphene stacks along the c-axis, and the ratio I2D/IG is less than 1 for all repeated laser writing samples, which implies the existence of multilayered graphene. ${ }^{[44]}$ Using Raman spectroscopy results and Equation $1^{[6]}$, the crystalline size of LIG, La, can also be estimated::

$$
L_{a}=\left(2.4 \times 10^{-10}\right) \times\left(\frac{I_{D}}{I_{G}}\right)^{-1} \times \lambda_{l,}^{4}
$$

b)
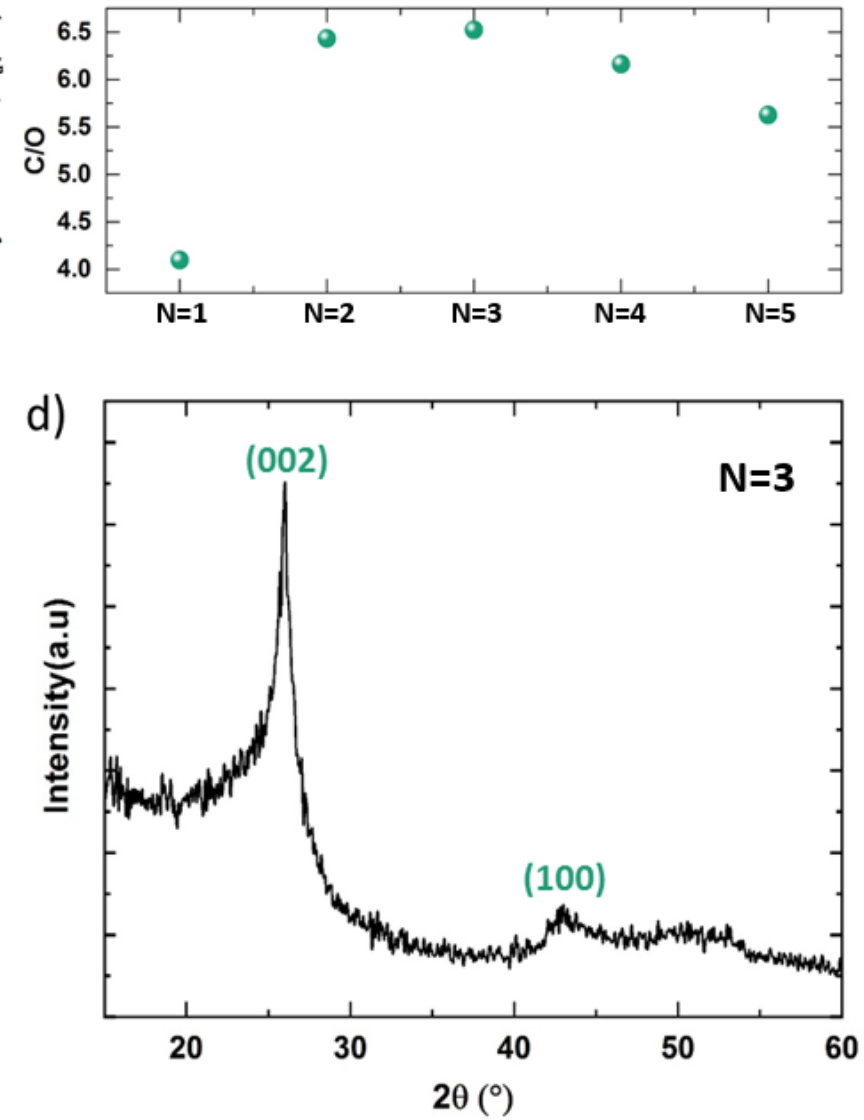

Fig 3. a) The $G$ and $D$ peak intensity ratios and average domain size as a function of repeated laser writing; b) $C / O$ ratio as a function of repeated laser writing; c) High-resolution XPS spectrum of the C-1s region of LIG after three times laser writing. d)XRD of powdered LIG scrapped from $\mathrm{PI}$ after three times of laser writing. 


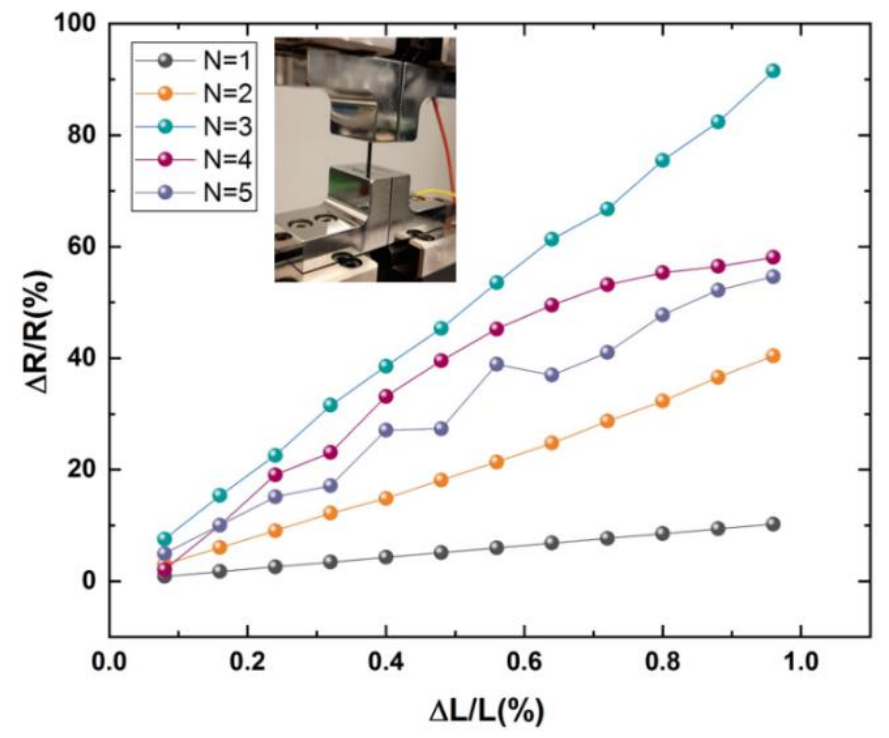

Fig. 4. The induced strain as a function of resistance change of LIG at different laser stages.

where $\lambda_{1}$ is the wavelength of the Raman laser $\left(\lambda_{1}=514\right.$ $\mathrm{nm})$. The $\mathrm{L}_{\mathrm{a}}$ values reach $\sim 60 \mathrm{~nm}$ after three laser writing steps (Fig. 3a). A further increase in the number of laser writing steps degrades the quality of the LIG with a $L_{a}$ of $21 \mathrm{~nm}$, which is attributed to the broken LIG bonds and partial oxidation of LIG in the air. XPS was used to examine the elemental composition of the LIG after each repeated laser writing step. XPS spectra of samples showed the signature of the major elements carbon (from the aryl group) and oxygen (from the ketone and ether bonds) only, as shown in Fig. S1. The $\mathrm{C} / \mathrm{O}$ ratio has increased by $\sim 37 \%$ after three laser writing steps, indicated an increased amount of carbon and decreased oxygen elements. Fig.3c and $\mathrm{d}$ show a high-resolution $\mathrm{C}-1 \mathrm{~s}$ peak of the XPS spectrum and an XRD pattern for LIG after three times of laser writing, respectively. Deconvoluted C-1s peak shows a prominent $\mathrm{C}-\mathrm{C}(284.8 \mathrm{eV})$ peak with considerably reduced $\mathrm{C}-\mathrm{O}(285.4 \mathrm{eV}), \quad \mathrm{C}=\mathrm{O}(286.2 \mathrm{eV})$ peaks, suggesting the predominance of $\mathrm{sp} 2$ carbons agreeing well with Raman spectra results. ${ }^{[4]}$ Meanwhile, XRD of powdered LIG shows a peak centered at $2 \theta=26.02^{\circ}$, and $2 \theta=42.9^{\circ}$, both of each correspond to previously reported LIG. ${ }^{[6,46]}$ The interlayer spacing of $0.342 \mathrm{~nm}$ between $(002)$ planes is derived using Bragg's equation (see Supporting Information), suggesting a high degree of graphitization. ${ }^{[6]}$

\section{RESULTS}

\section{A. Electromechanical performance}

The effect of multiple laser writing on the electromechanical parameters of the LIG has been evaluated by scribing the LIG sensor in the shape of a strip with a length of $20 \mathrm{~mm}$ while maintaining the same lasing power and speed as described in Section 2.1. The width and thickness of LIG increased by $12 \%$ and $33 \%$, respectively,
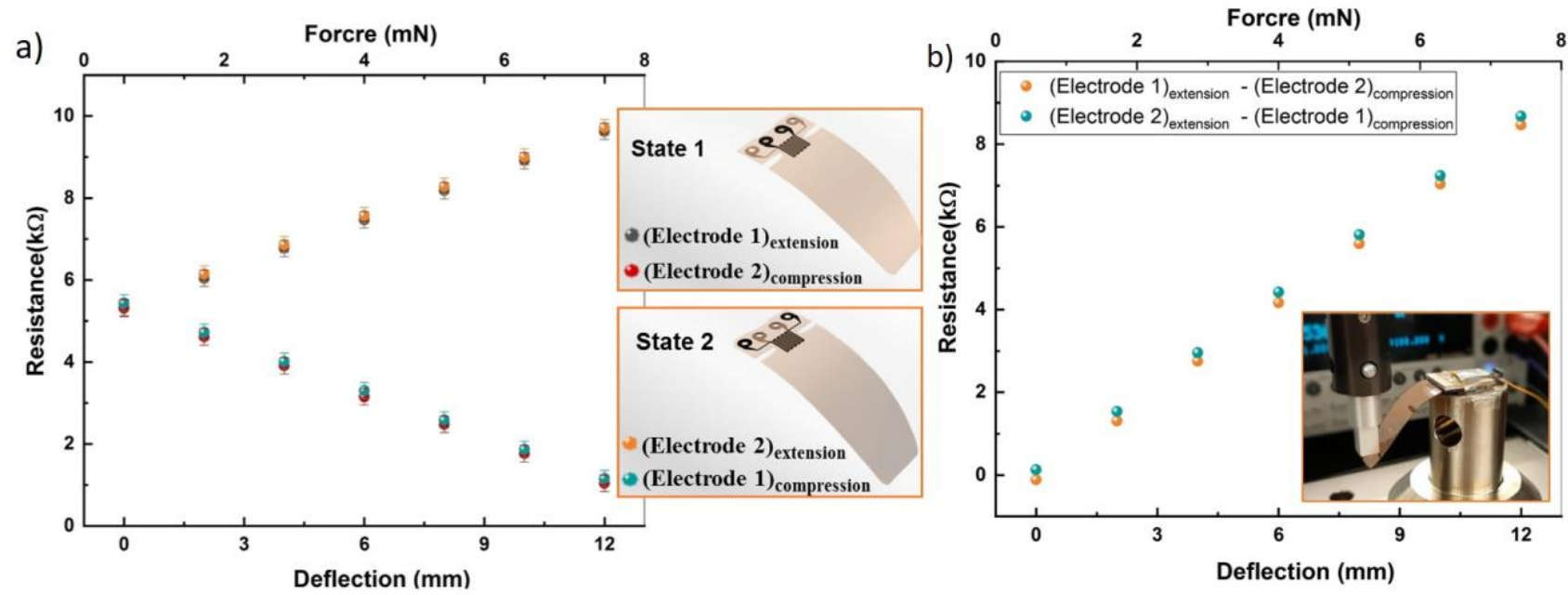

Fig. 5. a) The responses of one time $(\mathrm{N}=1)$ and three times laser scribed $(\mathrm{N}=3)$, double-sided $\mathrm{LIG}$ sensors to bending under extension and compression states. b) The resultant difference measurements of three times laser scribed sensor. 
after three times laser writing (Table S1). This increase in cross-section area could mean less electrical resistance for the same piece of LIG. However, the increased resistance and resistivity observed after each lasing step suggests the dominance of the effect of increased porosity. ${ }^{[47-49]} \mathrm{A}$ typical measure utilized to characterize the electromechanical performance of strain sensors is the Gauge factor (GF),

$$
G F=\frac{d R / R}{d l / l}=\frac{d \rho / \rho}{\varepsilon}+1+2 v
$$

where $\Delta \mathrm{l} / \mathrm{l}$ is the strain, $\mathrm{R}$ is the resistance without any strain, $\rho$ is the electrical resistivity, and $v$ is the Poisson ratio $\left(v_{\text {Kapton }}=0.34\right)$. The LIG electrode in the form of a strip with a length of $20 \mathrm{~mm}$ and a width of $1 \mathrm{~mm}$ was firmly positioned in the sample holder of an electromechanical tensile testing machine (5900-Series, Instron.) to apply strain (inset of Fig. 4). A DC current of $1 \mathrm{~mA}$ was applied to the LIG strain gauge to detect the variation of the electrical resistance during the tensile test via Keithley 2400 sourcemeter controlled with LabView software in a two probe setup. Fig. 4 shows a linear relationship between strain and relative change in resistance for all LIG samples, which is attributed to a narrowed and elongated conductive porous structure. The highest $\mathrm{GF} \simeq 91.2$ (Young modulus $\simeq 2.2 \mathrm{GPa}$, Yield Strength $\simeq 80 \mathrm{MPa}$ ) was achieved with LIG scribed by using three times laser writing, which is $\sim 720 \%$ higher than previously reported LIG strain gauges (Fig.S2). The increased GF is attributed to more polyporous and looser surface morphologies that provide for more displacement and the overlapping area of the graphene layers. A positive piezoresistivity was determined by an intrinsic contribution to the piezoresistivity $((\mathrm{d} \rho / \rho) / \mathrm{d} \mathrm{l} / \mathrm{l}=89.52)$.

\section{B. Force, deflection, and airflow detection.}

The following experiments were carried out with the LIG sensor realized using three times laser writing. The electromechanical tensile testing machine was also utilized to provide deflection in both bending directions, as shown in the insets of Fig. 5a. As expected, the resistance changes linearly with external force and deflection during sensor extension (LIG on the convex side). The electrical resistance of the LIG sensor scribed three times rises at the rate of $557 \Omega / \mathrm{mN}$ and $358 \Omega / \mathrm{mm}$ in ambient conditions (Fig. 5a). Meanwhile, the LIG bending sensor exhibits reducing resistance during compression (LIG on the concave side), decreasing at the rate of $-554 \Omega / \mathrm{mN},-356$ $\Omega / \mathrm{mm}$. In comparison to the sensor that is laser scribed once $(38 \Omega / \mathrm{mN}, 24 \Omega / \mathrm{mm}$ ), the sensor that was laser scribed three times showed $\sim 15$ times higher sensitivities in both directions. It was found that the resistance of LIG electrodes reduces by $4 \%$ over a temperature range of 20 $60^{\circ} \mathrm{C} .{ }^{[5,50,51]}$ Full temperature compensation was recently obtained by scribing the LIG patterns on both faces of the substrate and utilizing a difference measurement. ${ }^{[2]}$

In this study, the double-sided sensor configuration also provided an increased sensor sensitivity of $1108 \Omega / \mathrm{mN}$ and $712 \Omega / \mathrm{mm}$ with zero output voltage under no-load condition (Fig. 5b), as well as a rejection of common input signals, such as temperature. To examine the output of a LIG bending sensor to various airflow speeds, a setup was built wherein an air streaming in the tube induced sensor bending towards the direction of airflow (Fig. 6a). The airflow was quantified by bending induced from the drag associated with the airflow. The flow tube was constructed from obscure polyvinyl-chloride with a length of $0.25 \mathrm{~m}$ and a diameter of $50 \mathrm{~mm}$. An adjustable-speed electric fan with a brushless DC motor (KV 2600, Dynam RC Planes) was used for the regulation of the flow up to $12 \mathrm{~m} / \mathrm{s}$. The motor was connected to an electronic speed control driven by signals from the microcontroller (Atmega328, Microchip Inc.) and powered by a Switch Mode Power Supply (S1000-12, Festnight Inc.) A forward-curved fan,
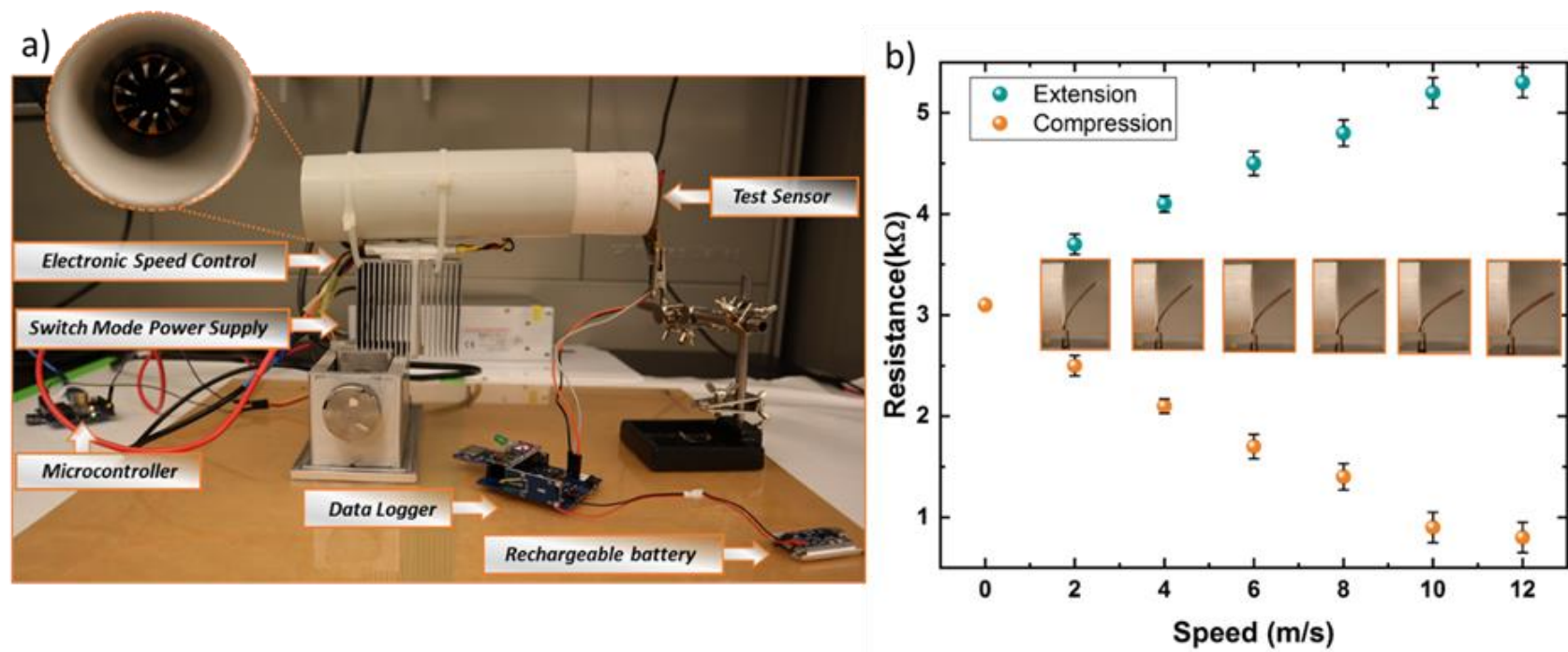

Fig. 6. a) Schematic of the flow test setup. The inset shows the speed-adjustable electric fan; b) Resistance measurements of LIG electrodes scribed on one side of the PI, in extension and compression states 
which is well suitable for the movement of large volumes of air against comparatively low pressures, was fitted into the pipe, as shown in the inset of Fig. 6a. The bending curvatures of the LIG sensor induced by various speeds of airflow are shown in the inset of Fig. 6b. The sensor showed a linear response within the speed range of 2-10 m/ with a sensitivity of $210 \Omega /(\mathrm{m} / \mathrm{s})$ for both extension and compression states. It should be noted that the sensor output varies depending on the length and cross-sectional area of the sensor substrate as well as the length of the piezoresistor. Depending on the application, the sensitivity can be increased using shorter piezoresistive electrodes ( Lpr $<5 \mathrm{~mm}$ ), thinner or wider substrates and decreased by the opposite measures(in case of the length see Fig.S3). consecutive times on PI with the geometry described in Section 2.1. A Parylene-C coating of $\sim 1 \mu \mathrm{m}$ in thickness was applied to the surface of porous graphene to protect it from adverse environmental impacts, such as rain. As seen in the inset of Fig.7a, the scribed circuit board features vias for stable and reliable electrical connection to the sensor and an operational amplifier (LM 324, ON Semiconductor, Inc.) for signal conditioning. Fig. 7a shows a photograph of the deployed quadcopter system and LIG sensor module attached to the front side of its frame (F450, DJI) using double-sided mounting tape (FK-M241, Fantastick). The drone flight controller included preinstalled open-source autopilot firmware (4Mini, Pixhawk 4 Inc.).The companion single-board computer (XU4, Odroid Inc. ) was connected to the flight controller using a serial connection. The package installed on the companion computer provided a driver for the autopilot with a communication protocol. The package published all estimated data into objects known as a)

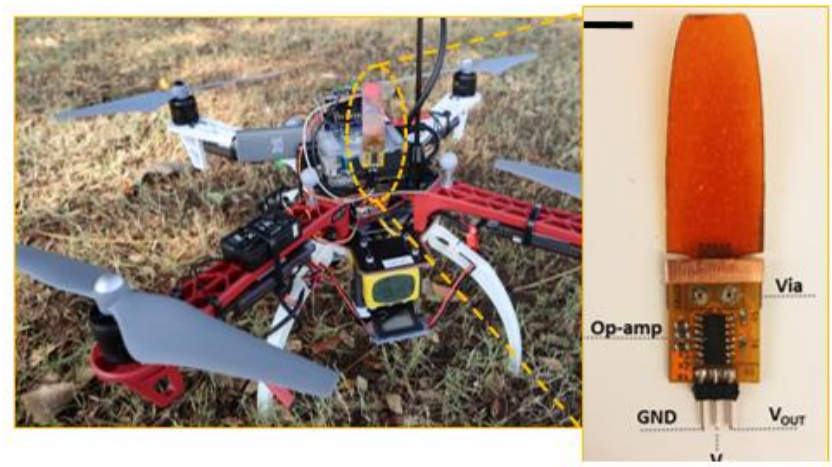

b)

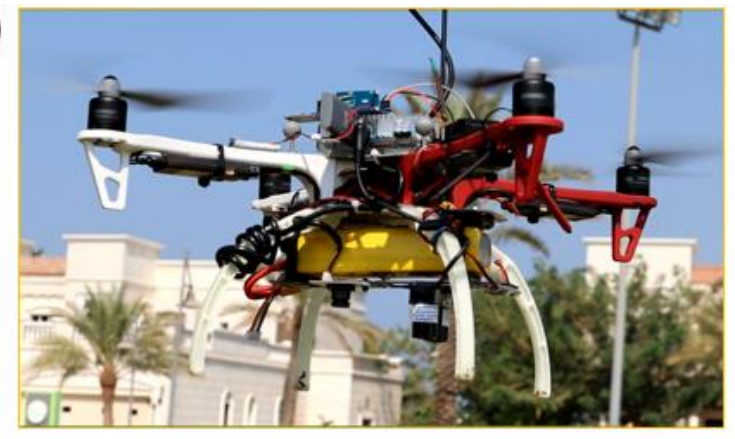

c)

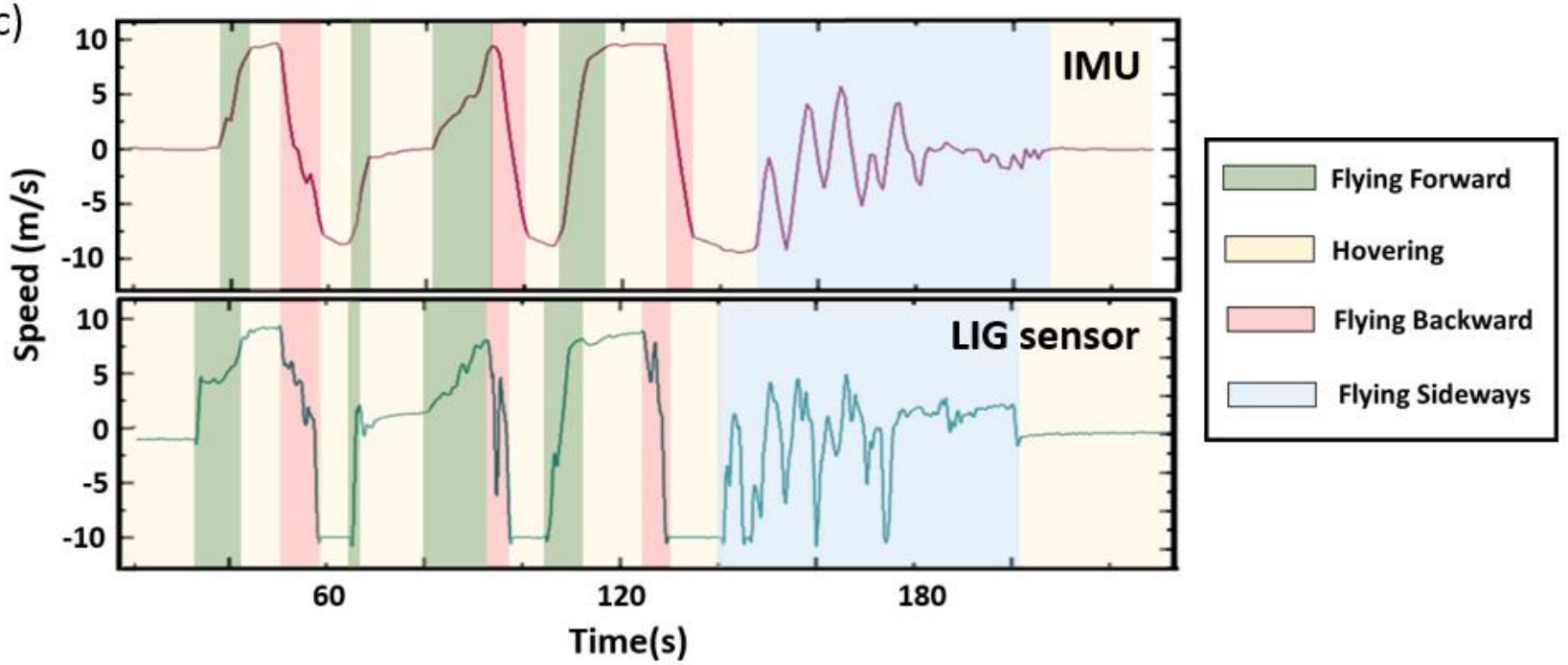

Fig. 7. a) LIG bending sensor integrated with PCB (scale bar: $1 \mathrm{~cm}$ ) and the in-house made drone; b) Sensor deployment in an outdoor environment to measure the velocity of the drone. c) The reference data (top) and LIG sensor data (bottom) corresponding velocity of

\section{UAV VELOCITY MONITORING}

The LIG bending sensor proved as a valuable instrument when combined with a ready-to-attach PCB assembly for easy integration into various applications. In order to monitor the speed and flying direction of an in-house made UAV/quadcopter drone, LIG electrodes were scribed three topics for unidirectional, streaming communication, which could be easily accessed and recorded with timestamps. The autopilot firmware provided linear and angular velocities, which were recorded in real-time using the Robot Operating System (ROS) tool into a file container. During the experiment, the UAV was controlled by the human in the Position Mode, which means that the UAV 
could actively brake level while being locked to a 3D spatial position. The signals of the LIG sensor were recorded during drone flying sessions in an outdoor environment (Fig. 7b) and calibrated to flying speeds through characterization obtained from Fig. 6b. Different maneuvers, such as flying forward, backward, hovering, and flying sideways, were carried out by the drone in 3.5 minutes. As it can be seen in Fig.7c, these maneuvers were explicitly reflected in the sensor and IMU responses with the highest speed of $\sim 10 \mathrm{~m} / \mathrm{s}$. To compare the sensor data with the signal provided by the IMU, both signals were sampled equidistantly and synchronously (Fig. 7c). The comparison was made through commonly used correlation, and root mean squared error (RMSE) analysis. The obtained Pearson coefficient of $r=0.91$ indicates a strong positive correlation between the two signals. Meanwhile, a large root mean squared error (RMSE) of $3.61 \mathrm{~m} / \mathrm{s}$ is attributed to the integration of the accumulated errors over time by the IMU system, as well as random vibrations of the LIG sensor due to orthogonal turbulent flows generated by UAV propellers. The effect of the latter could be considerably minimized using low-cost wind shielding at two sides of the sensor.

\section{CONCLUSION}

Enhanced properties of LIG sensors can open new perspectives for high-performance bending sensors capable of measuring flow, deflection, or force. A method of repeated laser writing was utilized to increase the porosity and uniform carbonization degree of LIG. Employing three times consecutive laser writing, several advantages arose. First, a decreased ID/IG ratio (by 67\%) indicated a reduction of structural defects and lower edge densities. Second, more polyporous and looser surface morphologies of LIG and reduced weight (by 35\%) were obtained, which provided more displacement and the overlapping area of the graphene layers. This allowed us to achieve a maximum GF of 91.2 with a piezoresistive coefficient of 89.5 , which is $720 \%$ times higher than that of previously reported LIG strain sensors. Third, increased crystalline size of the porous graphene ( $\sim 60 \mathrm{~nm})$ was obtained, which is two times higher than the one of the originally reported LIG by Lin et al. These results were verified via the elemental composition of LIG, which showed an increase of the $\mathrm{C} / \mathrm{O}$ ratio by $37 \%$. Meanwhile, the interlayer spacing of $0.342 \mathrm{~nm}$ between (002) planes, derived via Bragg's equation, suggested a high degree of graphitization. A homogeneous bidirectional response was obtained by scribing the electrodes on both faces of the substrate. Parylene-C coated LIG sensors were packaged to a ready-to-attach PCB assembly for easy integration into various applications. To this end, a LIG bending sensor was tested on a drone in an outdoor environment and performed very well as a cost-effective solution to provide absolute velocity up-dates.

\section{ACKNOWLEDGMENT}

This research is a contribution to the CAASE project funded by King Abdullah University of Science and Technology (KAUST) under the KAUST Sensor Initiative.

\section{REFERENCES}

[1] S. Luo, P. T. Hoang, T. Liu, Carbon 2016, 96, 522.

[2] A. Kaidarova, M. A. Khan, M. Marengo, L. Swanepoel, A. Przybysz, C. Muller, A. Fahlman, U. Buttner, N. R. Geraldi, R. P. Wilson, npj Flexible Electronics 2019, 3, 1.

[3] A. Kaidarova, N. Alsharif, B. N. M. Oliveira, M. Marengo, N. R. Geraldi, C. M. Duarte, J. Kosel, Global Challenges 2020, 2000001.

[4] X. Chen, F. Luo, M. Yuan, D. Xie, L. Shen, K. Zheng, Z. Wang, X. Li, L. Q. Tao, Advanced Functional Materials 2019 , 29, 1904706

[5] A. Kaidarova, J. Kosel, IEEE Sensors Journal 2020.

[6] J. Lin, Z. Peng, Y. Liu, F. Ruiz-Zepeda, R. Ye, E. L. Samuel, M. J. Yacaman, B. I. Yakobson, J. M. Tour, Nature communications 2014, 5, 1 .

[7] M. A. Khan, I. R. Hristovski, G. Marinaro, J. Kosel, IEEE Transactions on Magnetics 2017, 53, 1 .

[8] C. M. Tittle, D. Yilman, M. A. Pope, C. J. Backhouse, Advanced Materials Technologies 2018, 3, 1700207.

[9] X. Ye, J. Long, Z. Lin, H. Zhang, H. Zhu, M. Zhong, Carbon $2014,68,784$.

[10] M. G. Stanford, J. T. Li, Y. Chen, E. A. McHugh, A. Liopo, H. Xiao, J. M. Tour, ACS nano 2019, 13, 11912.

[11] R. Rahimi, M. Ochoa, W. Yu, B. Ziaie, ACS applied materials \& interfaces 2015, 7, 4463.

[12] Y. Wu, I. Karakurt, L. Beker, Y. Kubota, R. Xu, K. Y. Ho, S Zhao, J. Zhong, M. Zhang, X. Wang, Sensors and Actuators A: Physical 2018, 279, 46.

[13] A. Dallinger, K. Keller, H. Fitzek, F. Greco, ACS Applied Materials \& Interfaces 2020, 12, 19855

[14] M. G. Stanford, K. Yang, Y. Chyan, C. Kittrell, J. M. Tour, ACS nano 2019, 13, 3474

[15] L. Yang, N. Yi, J. Zhu, Z. Cheng, X. Yin, X. Zhang, H. Zhu, H. Cheng, Journal of Materials Chemistry A 2020, 8, 6487.

[16] J. C. Gerringer, A. G. Moran, T. Habib, M. J. Pospisil, J. H. Oh, B. R. Teipel, M. J. Green, ACS Applied Nano Materials 2019, 2, 7032

[17] Z. Wang, G. Wang, W. Liu, B. Hu, J. Liu, Y. Zhang, JOSA B 2020, 37, 546.

[18] G. Li, W.-C. Law, K. C. Chan, Green Chemistry 2018, 20 , 3689.

[19] R. K. Biswas, N. Farid, G. O'Connor, P. Scully, Journal of Materials Chemistry C 2020, 8, 4493.

[20] M. R. Bobinger, F. J. Romero, A. Salinas-Castillo, M. Becherer, P. Lugli, D. P. Morales, N. Rodríguez, A Rivadeneyra, Carbon 2019, 144, 116.

[21] D. X. Luong, K. Yang, J. Yoon, S. P. Singh, T. Wang, C. J. Arnusch, J. M. Tour, ACS nano 2019, 13, 2579.

[22] W. Song, J. Zhu, B. Gan, S. Zhao, H. Wang, C. Li, J. Wang, Small 2018, 14, 1702249.

[23] L. Huang, H. Wang, P. Wu, W. Huang, W. Gao, F. Fang, N. Cai, R. Chen, Z. Zhu, Sensors 2020, 20, 4266.

[24] Q. Tian, W. Yan, Y. Li, D. Ho, ACS applied materials \& interfaces 2020, 12, 9710.

[25] Y. Li, S. Zahran, Y. Zhuang, Z. Gao, Y. Luo, Z. He, L. Pei, R. Chen, N. El-Sheimy, Remote Sensing 2019, 11, 838.

J. C. Chow, J. D. Hol, H. Luinge, Drones 2018, 2, 6 . S. Wang, Z. Deng, G. Yin, Sensors 2016, 16, 280

Y. Zhao, IEEE Transactions on Vehicular Technology 2016, 66, 992.

A. El-Mowafy, N. Kubo, IET Intelligent Transport Systems 2018, 12, 901 .

[30] L. Wang, B. Song, X. Han, Y. Hao, Mathematical Problems in Engineering 2017, 2017.

[31] W. Youn, S. A. Gadsden, IEEE Access 2019, 7, 148989. 
[32] R. T. Casey, UC Santa Cruz, 2012

[33] J. Tiemann, F. Schweikowski, C. Wietfeld, "Design of an UWB indoor-positioning system for UAV navigation in GNSS-denied environments", presented at 2015 International Conference on Indoor Positioning and Indoor Navigation (IPIN), 2015.

[34] S. Godha, G. Lachapelle, M. E. Cannon, "Integrated GPS/INS system for pedestrian navigation in a signal degraded environment", presented at Ion gnss, 2006.

[35] S. Szabó, P. Enyedi, M. Horváth, Z. Kovács, P. Burai, T Csoknyai, G. Szabó, Journal of Cleaner Production 2016, 112 , 3820

[36] S. Zahran, A. M. Moussa, A. B. Sesay, N. El-Sheimy, IEEE Sensors Journal 2018, 19, 3067

[37] R. Ye, D. K. James, J. M. Tour, Advanced Materials 2019, 31, 1803621.

[38] R. Ye, D. K. James, J. M. Tour, Accounts of chemical research 2018, 51, 1609.

[39] M. G. Stanford, J. T. Li, Y. Chyan, Z. Wang, W. Wang, J. M. Tour, ACS nano 2019, 13, 7166

[40] A. Vashisth, M. Kowalik, J. C. Gerringer, C. Ashraf, A. C. Van Duin, M. J. Green, ACS Applied Nano Materials 2020, 3, 1881.

[41] R. Ye, Y. Chyan, J. Zhang, Y. Li, X. Han, C. Kittrell, J. M. Tour, Advanced Materials 2017, 29, 1702211.

[42] A. F. Carvalho, A. J. Fernandes, C. Leitão, J. Deuermeier, A. C. Marques, R. Martins, E. Fortunato, F. M. Costa, Advanced Functional Materials 2018, 28, 1805271

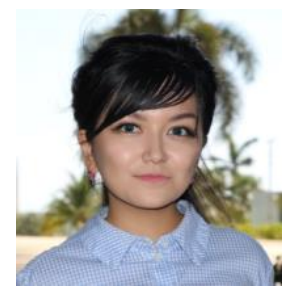

Altynay Kaidarova was born in Aksu, Pavlodar, Kazakhstan, in 1991. In 2010, she attained the Bolashak International Scholarship, which was awarded by the President of Kazakhstan. She had a foundation in Nanyang Technological University (NTU) in Singapore, 2011 and obtained first honor degree in Electronics and Communication Engineering from Liverpool University, Liverpool, England, in 2014. She received the M.S. in Electrical Engineering from King Abdullah University of Science and Technology (KAUST), Thuwal, Saudi Arabia in 2017. Currently, she is pursuing a Ph.D. degree in electrical engineering at KAUST. Her research interests are in the area of printed, wearable electronics and sensing systems.

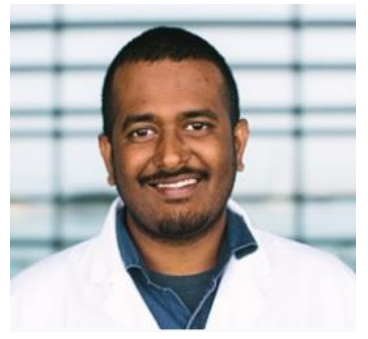

Mani Teja Vijjapu did his bachelors in Electronics and communications from Jawaharlal Technological University Anantapur, India in the year 2013. He worked as a research associate for Physical Research Laboratory, India for couple of years. He further continued to work as a research associate at IIT Madras, India. Later he pursued his masters in Solid state electronic devices from National Chiao-Tung University (NCTU), Taiwan and graduated in 2018. His Master's thesis is focused on fabricating a binary synapse like memristor using atomic layer deposition. He did his summer internship at National Device Laboratory (NDL-NARL Labs), Taiwan. He decided to pursue his passion being a PhD scholar at Sensors lab, KAUST from January 2018.

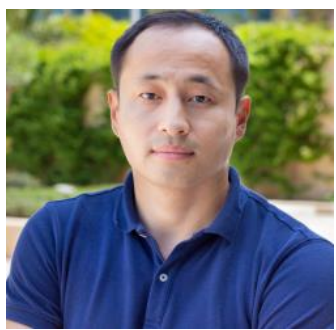

Kuat Telegenov received the BEng degree in Mechanical Engineering with a minor in Aeronautics from the University Technology of Malaysia, Malaysia, in 2012, and the M.Sc. degree in Mechanics from Gumilyov Eurasian National University, Kazakhstan, in 2014.Kuat Telegenov worked as an
[43] M. M. Lucchese, F. Stavale, E. M. Ferreira, C. Vilani, M. V. d. O. Moutinho, R. B. Capaz, C. A. Achete, A. Jorio, Carbon 2010, 48, 1592.

[44] S.-H. Lee, D. Kang, I.-K. Oh, Carbon 2017, 111, 248

[45] W. Zhang, Y. Lei, Q. Jiang, F. Ming, P. M. Costa, H. N Alshareef, Small Methods 2019, 3, 1900005.

[46] L. Li, J. Zhang, Z. Peng, Y. Li, C. Gao, Y. Ji, R. Ye, N. D. Kim, Q. Zhong, Y. Yang, Advanced Materials 2016, 28, 838.

[47] J.-C. Li, C.-H. Weng, F.-C. Tsai, W.-P. Shih, P.-Z. Chang, Applied Physics Letters 2016, 108, 013108.

[48] M. Hakamada, T. Kuromura, Y. Chen, H. Kusuda, M. Mabuchi, Materials transactions 2007, 48, 32.

[49] K. Verwer, G. P. Eberli, R. J. Weger, AAPG bulletin 2011, 95, 175 .

[50] A. Kaidarova, A. Almoudi, R. M. Allagani, M. Marengo, M. A. Khan, U. Buttner, C. M. Duarte, J. Kosel, "Flexible and Multi-functional Graphene sensor platform", presented at 2019 IEEE International Conference on Flexible and Printable Sensors and Systems (FLEPS), 2019.

[51] M. Marengo, G. Marinaro, J. Kosel, "Flexible temperature and flow sensor from laser-induced graphene", presented at 2017 IEEE SENSORS, 2017.

instructor in the School of Science and Technology at Nazarbayev University where he was teaching Machine Design, Computer-Aided Drawing (CAD) and Lab Machining courses. Prior to that, he worked as a research assistant at Nazarbayev University Research and Innovation System where he was working on design and control of underactuated robotic systems.

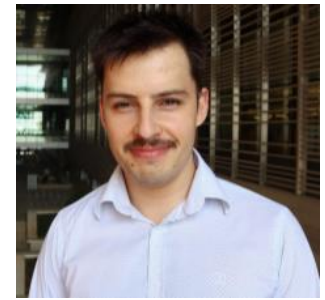

Alexander Przybysz is a scientific assistant and valuable member of Sensing, Magnetism, and Microsystems (SMM) Research group. Alexander's research interests include integrated circuits, microfabrication, and sensors. He is currently developing a reliable Cellular network, Marine Sensor Buoy to study marine environments.

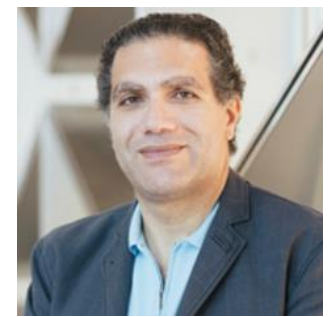

Khaled Nabil Salama (Senior Member, IEEE) received the B.S. degree (Hons.) from the Department of Electronics and Communications, Cairo University, Cairo, Egypt, in 1997, and the M.S. and Ph.D. degrees from the Department of Electrical Engineering, Stanford University, Stanford, CA, USA, in 2000 and 2005, respectively.,He was an Assistant Professor with the Rensselaer Polytechnic Institute, Troy, NY, USA, from 2005 to 2009. In 2009, he joined the King Abdullah University of Science and Technology, Thuwal, Saudi Arabia, where was the Founding Program Chair until 2011. He is currently a Professor with the King Abdullah University of Science and Technology. He has authored 225 articles and holds 14 patents on low-power mixed-signal circuits for intelligent fully integrated sensors and nonlinear electronics, in particular memristor devices. His research on CMOS sensors for molecular detection has been funded by the National Institutes of Health and the Defense Advanced Research Projects Agency.,Dr. Salama was a recipient of the Stanford-Berkeley Innovators Challenge Award in biological science. 


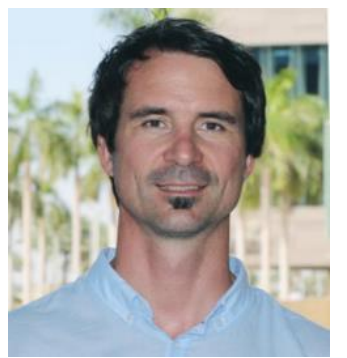

Jurgen Kosel (Senior Member, IEEE) received the Dipl.-Ing. (M.Sc.) and Ph.D. degrees in electrical engineering from the Vienna University of Technology, Vienna, Austria, in 2002 and 2006, respectively. From 2006 to 2007, he worked in the automotive industry as a Project Manager with Magna Powertrain, Graz, Austria. He was a Post-Doctoral Fellow at the Biomedical Engineering Research Group, Stellenbosch University, South Africa, from 2007 to 2009. Currently, he is an Adjunct Associate Professor of Electrical Engineering at the King Abdullah University of Science and Technology (KAUST), Thuwal, Saudi Arabia. He is the Head of Research Unit Sensor Applications, Silicon Austria Labs (SAL), Graz, Austria. His research interests are in the fields of micro and nano devices with a focus on magnetic transducers 